\title{
AUTOMORPHISMS OF THE UHF ALGEBRA THAT DO NOT EXTEND TO THE CUNTZ ALGEBRA
}

\author{
ROBERTO CONTI
}

(Received 23 March 2010; accepted 15 December 2010)

Communicated by G. A. Willis

\begin{abstract}
The automorphisms of the canonical core UHF subalgebra $\mathcal{F}_{n}$ of the Cuntz algebra $\mathcal{O}_{n}$ do not necessarily extend to automorphisms of $\mathcal{O}_{n}$. Simple examples are discussed within the family of infinite tensor products of (inner) automorphisms of the matrix algebras $M_{n}$. In that case, necessary and sufficient conditions for the extension property are presented. Also addressed is the problem of extending to $\mathcal{O}_{n}$ the automorphisms of the diagonal $\mathcal{D}_{n}$, which is a regular maximal abelian subalgebra with Cantor spectrum. In particular, it is shown that there exist product-type automorphisms of $\mathcal{D}_{n}$ that do not extend to (possibly proper) endomorphisms of $\mathcal{O}_{n}$.
\end{abstract}

2010 Mathematics subject classification: primary 46L40; secondary 46L05, 37B10.

Keywords and phrases: Cuntz algebra, endomorphism, automorphism, core UHF subalgebra, regular maximal abelian subalgebra.

\section{Introduction}

Among $C^{*}$ algebras, the class of uniformly hyperfinite (UHF) algebras is perhaps one of the first nontrivial (that is, infinite-dimensional) examples that comes to mind and it was investigated in depth in the outstanding work by Glimm in the early 1960s [9]. Since that time, many more types of $C^{*}$ algebras have been studied in depth, and our knowledge of the general theory of $C^{*}$ algebras has increased greatly. Some time later, the Cuntz algebras $\mathcal{O}_{n}$ made their appearance [5]. These $C^{*}$ algebras are quite different from UHF algebras as, for instance, they are traceless and purely infinite. However, it is well known that $\mathcal{O}_{n}$ contains canonically a copy of the UHF algebra of type $n^{\infty}$ (that is, the $C^{*}$-algebraic tensor product of countably infinitely many copies of $M_{n}$, the $n \times n$ matrix algebra over $\mathbb{C}$ ) as the canonical core UHF subalgebra $\mathcal{F}_{n}$.

In contrast to the case of $\mathcal{F}_{n}$, the study of automorphisms of $\mathcal{O}_{n}$ is quite challenging. Typical problems range from the construction of explicit examples to global properties of the automorphism group $\operatorname{Aut}\left(\mathcal{O}_{n}\right)$ and some notable subgroups and quotients.

(C) 2011 Australian Mathematical Publishing Association Inc. 1446-7887/2011 \$16.00 
The group $\operatorname{Aut}\left(\mathcal{O}_{n}, \mathcal{F}_{n}\right)$ of all automorphisms of $\mathcal{O}_{n}$ leaving $\mathcal{F}_{n}$ globally invariant has sporadically appeared in the literature [3, 4, 6]. In particular, it turns out that $\operatorname{Aut}\left(\mathcal{O}_{n}, \mathcal{F}_{n}\right)=\operatorname{Aut}\left(\mathcal{O}_{n}\right) \cap\left\{\lambda_{u} \mid u \in \mathcal{U}\left(\mathcal{F}_{n}\right)\right\}$ and so the examples of permutation automorphisms of $\mathcal{O}_{n}$ exhibited in [4] restrict to automorphisms of $\mathcal{F}_{n}$ that are also easily seen to be outer. In this short note, we will add another piece of information by showing that the canonical restriction map

$$
r: \operatorname{Aut}\left(\mathcal{O}_{n}, \mathcal{F}_{n}\right) \rightarrow \operatorname{Aut}\left(\mathcal{F}_{n}\right)
$$

is not surjective. The kernel of this group homomorphism consists of the gauge automorphism, by [3, Corollary 4.10]. A few remarks are in order. First, it is plain that all inner automorphisms of $\mathcal{F}_{n}$ extend to inner automorphisms of $\mathcal{O}_{n}$. Second, it is well known (see, for example, [7, Corollary IV.5.8]) that every automorphism of $\mathcal{F}_{n}$ is approximately inner in the sense that it is the limit of inner automorphisms of $\mathcal{F}_{n}$ in the topology of pointwise norm-convergence, that is, $\operatorname{Aut}\left(\mathcal{F}_{n}\right)=\overline{\operatorname{Inn}}\left(\mathcal{F}_{n}\right)$. Therefore our result, in particular, shows the existence of sequences of inner automorphisms of $\mathcal{O}_{n}$ that converge pointwise in norm on $\mathcal{F}_{n}$ but not on $\mathcal{O}_{n}$. Moreover, it makes it clear that there are strict inclusions (see [1])

$$
\operatorname{Inn}\left(\mathcal{F}_{n}\right) \subset r\left(\operatorname{Aut}\left(\mathcal{O}_{n}, \mathcal{F}_{n}\right)\right) \subset \operatorname{Aut}\left(\mathcal{F}_{n}\right) .
$$

Finally, we deduce that there are automorphisms of $\mathcal{F}_{n}$ that do not extend to $\mathcal{O}_{n}$ even as proper endomorphisms, as the latter possibility was ruled out in [3, Corollary 4.9]. In contrast, this last fact is not true for the canonical Cartan subalgebra $\mathcal{D}_{n}$ of $\mathcal{O}_{n}$ for there are proper endomorphisms of $\mathcal{O}_{n}$ that restrict to automorphisms of $\mathcal{D}_{n}$ (see [4]). Still, one can show the existence of automorphisms of $\mathcal{D}_{n}$ that do not extend to endomorphisms of $\mathcal{O}_{n}$.

We conclude this introductory section with a few words on notation (see [4]). For each integer $n \geq 2$, the Cuntz algebra $\mathcal{O}_{n}$ is the universal $C^{*}$ algebra generated by $n$ isometries $S_{1}, \ldots, S_{n}$ whose ranges sum to 1 . There is a one-to-one correspondence $v \mapsto \lambda_{v}$ between unitaries in $\mathcal{O}_{n}$ and unital *-endomorphisms of $\mathcal{O}_{n}$ that associates to $v \in \mathcal{U}\left(\mathcal{O}_{n}\right)$ the endomorphism $\lambda_{v}$ defined by $\lambda_{v}\left(S_{i}\right)=v S_{i}$ (where $\left.i=1, \ldots, n\right)$. The canonical core UHF subalgebra $\mathcal{F}_{n}$ is the norm-closure of the union of the matrix subalgebras $\mathcal{F}_{n}^{k} \simeq M_{n} \otimes \cdots \otimes M_{n}$ ( $k$ factors), where

$$
\mathcal{F}_{n}^{k}=\operatorname{span}\left\{S_{\alpha_{1}} \cdots S_{\alpha_{k}} S_{\beta_{k}}^{*} \ldots S_{\beta_{1}}^{*}, 1 \leq \alpha_{1}, \ldots, \alpha_{k}, \beta_{1}, \ldots, \beta_{k} \leq n\right\} .
$$

The UHF subalgebra $\mathcal{F}_{n}$ admits a unique normalized trace, denoted $\tau$. We also consider $\mathcal{D}_{n}$, the diagonal subalgebra of $\mathcal{F}_{n}$, which is equal to the norm-closure of the union of the commutative subalgebras $\mathcal{D}_{n}^{k}=\mathcal{D}_{n} \cap \mathcal{F}_{n}^{k} \simeq \mathbb{C}^{n^{k}}$. We define the canonical endomorphism $\varphi$ of $\mathcal{O}_{n}$ by $\varphi(x)=\sum_{i=1}^{n} S_{i} x S_{i}^{*}$ for all $x \in \mathcal{O}_{n}$. This shift endomorphism satisfies $S_{i} x=\varphi(x) S_{i}$ when $x \in \mathcal{O}_{n}$ and $i=1, \ldots, n$. For a unital $C^{*}$ algebra $B, \operatorname{Aut}(B)$ and $\operatorname{Inn}(B)$ are the groups of automorphisms and inner automorphisms of $B$, respectively. For unital $C^{*}$ algebras $A \subseteq B, \operatorname{Aut}(B, A)$ and $\operatorname{Aut}_{A}(B)$ are the groups of automorphisms of $B$ leaving $A$ globally and pointwise invariant, respectively. 


\section{Main result}

We refer to [8, Ch. VI] for generalities about UHF algebras and their automorphisms. To simplify the notation we will often represent elements of $\mathcal{F}_{n}$ by tensor products of matrices, through the canonical identification of $\mathcal{F}_{n}$ with $\bigotimes_{i=1}^{\infty} M_{n}$.

Given a sequence of unitaries $\underline{u}=\left(u_{i}\right)$ with $u_{i} \in \mathcal{U}\left(\mathcal{F}_{n}^{1}\right) \simeq U(n)$, we consider the associated automorphism $\alpha_{\underline{u}}$ of $\mathcal{F}_{n}$ such that

$$
\alpha_{\underline{u}}\left(S_{\alpha} S_{\beta}^{*}\right)=u_{1} S_{\alpha_{1}} u_{2} S_{\alpha_{2}} \cdots u_{k} S_{\alpha_{k}} S_{\beta_{k}}^{*} u_{k}^{*} \cdots S_{\beta_{2}}^{*} u_{2}^{*} S_{\beta_{1}}^{*} u_{1}^{*},
$$

for all multi-indices $\alpha=\left(\alpha_{1}, \ldots, \alpha_{k}\right)$ and $\beta=\left(\beta_{1}, \ldots, \beta_{k}\right)$ of the same length $k$ and for all $k \geq 1$. In the tensor product picture, $\alpha_{u}$ is nothing more than the infinite tensor product automorphism $\bigotimes_{i=1}^{\infty} \operatorname{Ad}\left(u_{i}\right)$. It is also clear that if

$$
\lim _{k \rightarrow \infty} u_{1} \otimes u_{2} \otimes \cdots \otimes u_{k} \otimes 1 \otimes 1 \otimes \cdots=: u
$$

exists in $\bigotimes_{i=1}^{\infty} M_{n}$ then $\bigotimes_{i=1}^{\infty} \operatorname{Ad}\left(u_{i}\right)=\operatorname{Ad}(u)$ is inner, while the converse holds true whenever $1 \in \sigma\left(u_{i}\right)$ for all $i$ (the latter assumption can always be satisfied by rotating the $u_{i}$ if necessary); see [8, Theorem 6.3].

Of course, if $\alpha_{\underline{u}}=\operatorname{Ad}(u)$ is inner then it extends to an inner automorphism $\lambda_{u \varphi\left(u^{*}\right)}$ of $\mathcal{O}_{n}$. It is quite possible that even though $\alpha_{\underline{u}}$ is outer, it still extends to an automorphism of $\mathcal{O}_{n}$. A simple example of this situation arises when $\underline{u}$ is a nonscalar constant sequence: $u_{1}=u_{2}=u_{3}=\cdots$. Then $\alpha_{\underline{u}}=\bigotimes_{i=1}^{\infty} \operatorname{Ad}\left(u_{1}\right)$ is an outer automorphism of $\mathcal{F}_{n}$ and extends to the (still outer) Bogolubov automorphism $\lambda_{u_{1}}$ of $\mathcal{O}_{n}$. At this point one could suspect that the possibility of extending $\alpha_{\underline{u}}$ to $\mathcal{O}_{n}$ depends on whether

$$
\lim _{k \rightarrow \infty} u_{1} \otimes u_{2} u_{1}^{*} \otimes u_{3} u_{2}^{*} \otimes \cdots \otimes u_{k+1} u_{k}^{*} \otimes 1 \otimes 1 \cdots
$$

exists in $\bigotimes_{i=1}^{\infty} M_{n}$. This is indeed the case, as we see from the following result.

THEOREM 2.1. Let $u_{1}, u_{2}, u_{3}, \ldots$ be an infinite sequence of unitaries in $\mathcal{U}\left(\mathcal{F}_{n}^{1}\right)$. If the limit in Equation (2.2) exists and thus defines a unitary element $v \in \mathcal{F}_{n}$, then $\lambda_{v}$ is an automorphism of $\mathcal{O}_{n}$ that, in restriction to $\mathcal{F}_{n}$, coincides with $\alpha_{\underline{u}}=\bigotimes_{i=1}^{\infty} \operatorname{Ad}\left(u_{i}\right)$. Conversely, suppose that $\alpha_{\underline{u}}$ extends to an endomorphism of $\mathcal{O}_{n}^{-}$. Then there are phases $\left(e^{i \theta_{k}}\right)_{k}$ such that the limit in Equation (2.2) exists for the sequence $\left(u_{k}^{\prime}\right)$, where $u_{k}^{\prime}=e^{i \theta_{k}} u_{k}$.

PROOF. If $v$ is defined by the limit as above, then one can easily check that $\lambda_{v}$ coincides with $\alpha_{\underline{u}}$ in restriction to $\mathcal{F}_{n}$, and thus $\lambda_{v} \in \operatorname{Aut}\left(\mathcal{O}_{n}\right)$ by [3, Corollary 4.9].

Conversely, let $\lambda_{w}$ be an endomorphism of $\mathcal{O}_{n}$ such that $\lambda_{w}(x)=\alpha_{\underline{u}}(x)$ for all $x \in \mathcal{F}_{n}$. Then $\lambda_{w} \in \operatorname{Aut}\left(\mathcal{O}_{n}\right)$ and $w \in \mathcal{F}_{n}$. Now,

$$
w S_{i} S_{j}^{*} w^{*}=u_{1} S_{i} S_{j}^{*} u_{1}^{*}
$$


for all $i, j=1, \ldots, n$ and thus $w^{*} u_{1} \in\left(\mathcal{F}_{n}^{1}\right)^{\prime} \cap \mathcal{F}_{n}=\varphi\left(\mathcal{F}_{n}\right)$, that is, $w=u_{1} \varphi\left(z_{1}\right)$ for some $z_{1} \in \mathcal{U}\left(\mathcal{F}_{n}\right)$. But then

$$
w \varphi(w) S_{i} S_{j} S_{k}^{*} S_{h}^{*} \varphi\left(w^{*}\right) w^{*}=u_{1} \varphi\left(u_{2}\right) S_{i} S_{j} S_{k}^{*} S_{h}^{*} \varphi\left(u_{2}^{*}\right) u_{1}^{*}
$$

for all $i, j, h, k=1, \ldots, n$, that is,

$$
\varphi\left(w^{*}\right) w^{*} u_{1} \varphi\left(u_{2}\right) \in\left(\mathcal{F}_{n}^{2}\right)^{\prime} \cap \mathcal{F}_{n}=\varphi^{2}\left(\mathcal{F}_{n}\right) .
$$

As argued above, this means that $w^{*} z_{1}^{*} u_{2}=\varphi\left(z_{1}^{*}\right) u_{1}^{*} z_{1}^{*} u_{2} \in \varphi\left(\mathcal{F}_{n}\right)$. Thus $u_{1}^{*} z_{1}^{*} u_{2}=$ $\varphi\left(z_{2}^{*}\right) \in \varphi\left(\mathcal{F}_{n}\right)$ for some unitary $z_{2} \in \mathcal{U}\left(\mathcal{F}_{n}\right)$ from which $z_{1}^{*}=u_{1} \varphi\left(z_{2}^{*}\right) u_{2}^{*}=\varphi\left(z_{2}^{*}\right) u_{1} u_{2}^{*}$ and consequently

$$
w=u_{1} \varphi\left(u_{2} u_{1}^{*}\right) \varphi^{2}\left(z_{2}\right) .
$$

Repeating this argument, one obtains that, for any positive integer $k$,

$$
w=u_{1} \varphi\left(u_{2} u_{1}^{*}\right) \varphi^{2}\left(u_{3} u_{2}^{*}\right) \cdots \varphi^{k}\left(u_{k+1} u_{k}^{*}\right) \varphi^{k+1}\left(z_{k+1}\right)
$$

for a suitable unitary $z_{k} \in \mathcal{F}_{n}$. Moreover, there is a sequence of phases $\left(e^{i \theta_{k}}\right)_{k}$ such that, after replacing $u_{k}$ with $e^{i \theta_{k}} u_{k}$, one can always assume that $\tau\left(z_{k}\right) \in \mathbb{R}_{+}$.

Consider now the $\tau$-invariant conditional expectation $E_{k}: \mathcal{F}_{n} \rightarrow \mathcal{F}_{n}^{k}$ defined by $E_{k}=\mathrm{id}_{k} \otimes \tau$, where $\mathrm{id}_{k}$ is the identity on $\mathcal{F}_{n}^{k}$. For this, $x=\lim _{k}\left(E_{k}(x)\right)$ for all $x \in \mathcal{F}_{n}$. As argued above, it is clear that $E_{k}(w)=u_{1} \otimes \cdots \otimes u_{k+1} u_{k}^{*} \tau\left(z_{k+1}\right)$ for all $k \geq 1$ and thus

$$
1=\|w\|=\lim _{k \rightarrow \infty}\left\|u_{1} \otimes \cdots \otimes u_{k+1} u_{k}^{*}\right\| \tau\left(z_{k+1}\right)=\lim _{k \rightarrow \infty} \tau\left(z_{k+1}\right)
$$

That is,

$$
\lim _{k \rightarrow \infty}\left\|w-u_{1} \otimes \cdots \otimes u_{k+1} u_{k}^{*}\right\|=\lim _{k \rightarrow \infty}\left\|w-u_{1} \otimes \cdots \otimes u_{k+1} u_{k}^{*} \tau\left(z_{k+1}\right)\right\|=0
$$

as required.

The automorphisms of $\mathcal{F}_{n}$ of the form $\alpha_{\underline{u}}$ satisfy $\alpha_{\underline{u}}\left(\mathcal{F}_{n}^{k}\right)=\mathcal{F}_{n}^{k}$ for all $k$. An endomorphism of the Cuntz algebra is said to be localized if it is induced by a unitary element of some finite matrix algebra, that is, it is in $\bigcup_{k=1}^{\infty} \mathcal{F}_{n}^{k}$, [2].

Corollary 2.2. Suppose that $\alpha_{\underline{u}}$ extends to an automorphism of the Cuntz algebra. Then the following conditions are equivalent:

(a) one extension of $\alpha_{\underline{u}}$ is localized;

(b) all extensions of $\alpha_{\underline{u}}$ are localized;

(c) eventually $u_{k+1} u_{k}^{*} \in \mathbb{T} 1$.

PROOF. This result follows from the fact that any two such extensions must differ by a gauge automorphism. Moreover, a unitary map implementing any of them is obtained through Equation (2.2) up to phases, as explained above. 


\section{Outlook}

We would like to mention a few related problems. On the one hand, one should investigate the detailed structure of $\operatorname{Aut}\left(\mathcal{O}_{n}, \mathcal{F}_{n}\right)$ and find an intrinsic characterization of automorphisms of $\mathcal{F}_{n}$ that extend to $\mathcal{O}_{n}$. On the other hand, one should study the analogous problems for the extension of automorphisms from the diagonal $\mathcal{D}_{n}$ to $\mathcal{F}_{n}$ and from $\mathcal{D}_{n}$ to $\mathcal{O}_{n}$.

In this respect, we can say something more about the extension of automorphisms from $\mathcal{D}_{n}$ to $\mathcal{O}_{n}$. In particular, we are now ready to show the existence of automorphisms of $\mathcal{D}_{n}$ that do not extend to (possibly proper) endomorphisms of $\mathcal{O}_{n}$. This will easily follow also from Theorem 3.3 below, but the following observation is more in line with the criterion given in Theorem 2.1.

PROPOSITION 3.1. Let $\alpha$ be a product type automorphism of the diagonal, that is, $\alpha\left(\varphi^{k}\left(\mathcal{D}_{n}^{1}\right)\right)=\varphi^{k}\left(\mathcal{D}_{n}^{1}\right)$ for all $k \geq 0$. Then $\alpha$ extends to a (possibly proper) endomorphism of $\mathcal{O}_{n}$ if and only if the action on each $\varphi^{k}\left(\mathcal{D}_{n}^{1}\right)$ is eventually identical. In that case, $\alpha$ extends to a permutation automorphism of $\mathcal{O}_{n}$.

PROOF. Suppose that $\alpha$ extends to an endomorphism $\lambda_{u}$ of $\mathcal{O}_{n}$. Then it follows that $\lambda_{u}\left(\varphi^{k}\left(\mathcal{D}_{n}^{1}\right)\right)=\varphi^{k}\left(\mathcal{D}_{n}^{1}\right)$ for all $k$ and an easy induction shows that $\operatorname{Ad}(u)$ preserves each $\varphi^{k}\left(\mathcal{D}_{n}^{1}\right)$. Thus, in particular, $u$ is in the normalizer of $\mathcal{D}_{n}$. Consequently (see [10]), we may write $u=w v$ where $w \in \mathcal{S}_{n}$ and $v \in \mathcal{U}\left(\mathcal{D}_{n}\right)$, where $\mathcal{S}_{n}$ is the subgroup of $\mathcal{U}\left(\mathcal{O}_{n}\right)$ of unitaries that can be written as finite sums of words in $S_{i}$ and $S_{i}^{*}$.

It now follows that the restriction $\alpha$ of $\lambda_{u}$ to $\mathcal{D}_{n}$ coincides with the restriction of $\lambda_{w}$ to $\mathcal{D}_{n}$. Thus $\operatorname{Ad}(w)$ preserves each $\varphi^{k}\left(\mathcal{D}_{n}^{1}\right)$ and so we may deduce that the restriction to $\mathcal{D}_{n}$ of the trace $\tau$ is $\operatorname{Ad}(w)$-invariant. However, this is only possible if $w$ belongs to $\mathcal{F}_{n}$, that is, if $w$ is a permutation matrix.

Since $\operatorname{Ad}(w)$ preserves each $\varphi^{k}\left(\mathcal{D}_{n}^{1}\right)$, it follows that

$$
w=w_{1} \varphi\left(w_{2}\right) \varphi^{2}\left(w_{3}\right) \cdots \varphi^{r}\left(w_{r+1}\right)
$$

for some positive integer $r$ and permutation matrices $w_{j}$ in $\mathcal{F}_{n}^{1}$ (where $j=1, \ldots, r$ ). This implies that the restriction of $\lambda_{w}$ to $\varphi^{k}\left(\mathcal{F}_{n}^{1}\right)$ coincides with the restriction of $\operatorname{Ad}\left(\varphi^{k}\left(w_{k+1} w_{k} \cdots w_{2} w_{1}\right)\right)$. This means that $\lambda_{w}$, in restriction to $\mathcal{F}_{n}$, is a product type automorphism of $\mathcal{F}_{n}$ for which the limit in Equation (2.2) is equal to 1 (actually the terms of the sequence eventually stabilize). Therefore, by Theorem $2.1, \lambda_{w}$ is (and thus $\alpha$ extends to) an automorphism of $\mathcal{O}_{n}$.

We denote by $\operatorname{EndAut}\left(\mathcal{O}_{n}, \mathcal{D}_{n}\right)$ the subsemigroup of $\operatorname{End}\left(\mathcal{O}_{n}\right)$ of those endomorphisms that restrict to automorphisms of $\mathcal{D}_{n}$ and by $\operatorname{End}_{\mathcal{D}_{n}}\left(\mathcal{O}_{n}\right)$ the subsemigroup of $\operatorname{EndAut}\left(\mathcal{O}_{n}, \mathcal{D}_{n}\right)$ of those endomorphisms acting trivially on $\mathcal{D}_{n}$. Then

$$
\operatorname{Aut}\left(\mathcal{O}_{n}, \mathcal{D}_{n}\right) \subset \operatorname{EndAut}\left(\mathcal{O}_{n}, \mathcal{D}_{n}\right)
$$

by the analysis in [4]. However, the following result is already implicit in [6]. 
PROPOSITION 3.2. With the notation above,

$$
\operatorname{Aut}_{\mathcal{D}_{n}}\left(\mathcal{O}_{n}\right)=\operatorname{End}_{\mathcal{D}_{n}}\left(\mathcal{O}_{n}\right)
$$

PROOF. Let $u$ be a unitary element of $\mathcal{O}_{n}$. If $\lambda_{u}(x)=x$ for all $x \in \mathcal{D}_{n}$ then it is easy to see by induction on $k$ that $u$ commutes with $\varphi^{k}\left(\mathcal{D}_{n}^{1}\right)$ for all $k \geq 1$, and therefore

$$
u \in\left(\bigcup_{k \geq 0} \varphi^{k}\left(\mathcal{D}_{n}^{1}\right)\right)^{\prime} \cap \mathcal{O}_{n}=\mathcal{D}_{n}^{\prime} \cap \mathcal{O}_{n} .
$$

Since the diagonal $\mathcal{D}_{n}$ is a maximal abelian subalgebra of $\mathcal{O}_{n}$, it follows that $u \in \mathcal{D}_{n}$ and therefore $\lambda_{u} \in \operatorname{Aut}_{\mathcal{D}_{n}}\left(\mathcal{O}_{n}\right)$ by [6].

THEOREM 3.3. The restriction map

$$
r: \operatorname{EndAut}\left(\mathcal{O}_{n}, \mathcal{D}_{n}\right) \rightarrow \operatorname{Aut}\left(\mathcal{D}_{n}\right)
$$

is not surjective. Furthermore, $r\left(\operatorname{EndAut}\left(\mathcal{O}_{n}, \mathcal{D}_{n}\right)\right)$ is not a subgroup of $\operatorname{Aut}\left(\mathcal{D}_{n}\right)$. Indeed, it is the disjoint union of the subgroup of those automorphisms that extend to automorphisms of $\mathcal{O}_{n}$, and the subsemigroup of those automorphisms that extend to proper endomorphisms of $\mathcal{O}_{n}$.

PROOF. The first statement follows from Proposition 3.1.

Let $u$ be a unitary element of $\mathcal{O}_{n}$ such that $\lambda_{u}$ is a proper endomorphism of $\mathcal{O}_{n}$ and $\lambda_{u}\left(\mathcal{D}_{n}\right)=\mathcal{D}_{n}$ (see [4]). We claim that $\left(\left.\lambda_{u}\right|_{\mathcal{D}_{n}}\right)^{-1} \in \operatorname{Aut}\left(\mathcal{D}_{n}\right)$ does not extend to an endomorphism of $\mathcal{O}_{n}$. For otherwise, let $\lambda_{v}$ be such an extension. Then $\lambda_{v} \lambda_{u}(x)=x$ for all $x \in \mathcal{D}_{n}$ and thus $\lambda_{v} \lambda_{u}$ is an automorphism of $\mathcal{O}_{n}$, by Proposition 3.2, which is a contradiction. A similar argument shows that if two endomorphisms $\lambda_{u_{1}}$ and $\lambda_{u_{2}}$ of $\mathcal{O}_{n}$ restrict to the same automorphism of $\mathcal{D}_{n}$ then they are either both automorphisms or both proper endomorphisms.

From this discussion, the automorphisms of $\mathcal{D}_{n}$ obtained by restriction of proper endomorphisms of $\mathcal{O}_{n}$ are necessarily not of product type.

Finally, we observe that the subgroup $r\left(\operatorname{Aut}\left(\mathcal{O}_{n}, \mathcal{D}_{n}\right)\right)$ is not normal in $\operatorname{Aut}\left(\mathcal{D}_{n}\right)$. Indeed, by Gelfand duality, $\operatorname{Aut}\left(\mathcal{D}_{n}\right) \simeq \operatorname{Homeo}(\mathcal{C})$, where $\mathcal{C}$ is the Cantor set, and the latter group is known to be simple.

\section{References}

[1] R. J. Archbold, 'On the "flip-flop" automorphism of $C^{*}\left(S_{1}, S_{2}\right)$ ', Q. J. Math. 30 (1979), 129-132.

[2] R. Conti and C. Pinzari, 'Remarks on endomorphisms of Cuntz algebras', J. Funct. Anal. 142 (1996), 369-405.

[3] R. Conti, M. Rørdam and W. Szymański, 'Endomorphisms of $\mathcal{O}_{n}$ which preserve the canonical UHF-subalgebra', J. Funct. Anal. 259 (2010), 602-617.

[4] R. Conti and W. Szymański, 'Labeled trees and localized automorphisms of the Cuntz algebras', Trans. Amer. Math. Soc., arXiv:0805.4654, to appear.

[5] J. Cuntz, 'Simple $C^{*}$-algebras generated by isometries', Comm. Math. Phys. 57 (1977), 173-185. 
[6] J. Cuntz, 'Automorphisms of certain simple $C^{*}$-algebras', in: Quantum Fields-Algebras, Processes (ed. L. Streit) (Springer, Vienna-New York, 1980).

[7] K. R. Davidson, C*-Algebras by Example, Fields Institute Monographs, 6 (American Mathematical Society, Providence, RI, 1996).

[8] D. E. Evans and Y. Kawahigashi, Quantum Symmetries on Operator Algebras, Oxford Mathematical Monographs (Oxford University Press, Oxford, 1998).

[9] J. G. Glimm, 'On a certain class of operator algebras', Trans. Amer. Math. Soc. 95 (1960), $318-340$.

[10] S. C. Power, 'Homology for operator algebras, III. Partial isometry homotopy and triangular algebras', New York J. Math. 4 (1998), 35-56.

ROBERTO CONTI, Dipartimento di Scienze,

Università di Chieti-Pescara 'G. D’Annunzio', Viale Pindaro 42,

I-65127 Pescara, Italy

e-mail: conti@sci.unich.it 